\begin{tabular}{ll} 
Abstract 204 Table 2 & Clinical characteristics ( $\mathrm{n}, \%)$ \\
\hline Muco-cutanous & $108(72 \%)$ \\
\hline Arthritis & $100(66.7 \%)$ \\
Renal disease & $72(48 \%)$ \\
Leukopenia/lymphopenia & $94(62.7 \%)$ \\
Hemolytic anaemia & $13(8.7 \%)$ \\
Thrombocytopenia & $28(18.7 \%)$ \\
Serositis & $30(20 \%)$ \\
Nervous system disease & $11(7.3 \%)$ \\
Anti-phospholipid Syndrome & $24(16 \%)$ \\
\hline
\end{tabular}

Abstract 204 Table 3 Treatment $(n, \%)$

\begin{tabular}{ll}
\hline Steroid/pulse treatment & $149(99.3 \%) / 38(25.3 \%)$ \\
\hline Hydroxycholoroquine & $150(100 \%)$ \\
Azathioprine & $107(71.3 \%)$ \\
Mycophenolate mofetil & $55(36.7 \%)$ \\
Cyclophosphamide (iv) & $45(30 \%) ; 10 \pm 4.5$ cycles \\
Rituximab & $15(10 \%)$ \\
Warfarin & $31(20.7 \%)$ \\
Intravenous immunglobulin (IVIG) & $3(2 \%)$ \\
Plasmapheresis & $2(1.3 \%)$ \\
\hline
\end{tabular}

hypertension, 21 (14\%) had avascular necrosis, 6 (4\%) had malignancy. SLE is an autoimmune disease requiring multi-faceted approach.

\section{ANNEXIN II-BINDING IMMUNOGLOBULIN G LEVEL CORRELATES WITH CLINICAL AND RENAL HISTOLOGICAL DISEASE ACTIVITY IN LUPUS NEPHRITIS}

KF Cheung*, S Yung, M Chau, DYH Yap, TM Chan. The University of Hong Kong, Medicine, Pokfulam, Hong Kong S.A.R

\subsection{6/lupus-2017-000215.205}

Background and aims Annexin II mediates anti-dsDNA antibody binding to mesangial cells and downstream inflammatory and fibrotic processes. We investigated the relationship between annexin II-binding IgG and clinical or histological activity in lupus nephritis.

Methods Serial serum samples from 28 patients with Class III/ $\mathrm{IV} \pm \mathrm{V}$ lupus nephritis were studied. Annexin II-binding IgG level was measured with an in-house ELISA. Glomeruli were isolated from NZBWF1 mice, gene and protein expression of annexin II and its binding protein p11 were investigated by real-time PCR and cytochemical staining respectively. Ultrastructural localization of annexin II was determined by electron microscopy and immunogold staining.

Results Annexin II-binding IgG level was associated with antidsDNA level and disease activity in $42 \%$ of lupus nephritis patients. Annexin II-binding IgG level correlated with Activity Index $(r=0.44, p=0.04)$, leukocyte infiltration score $(r=0.52$, $\mathrm{p}=0.02)$, and karyorrhexis/fibrinoid necrosis score $(\mathrm{r}=0.66$, $\mathrm{p}=0.002$ ) in renal biopsies, and also with the amount of mesangial electron-dense deposit scored semi-quantitatively $(\mathrm{r}=0.63, \mathrm{p}=0.009)$. Glomerular annexin II and $\mathrm{p} 11$ expression increased with disease progression in NZBWF1 mice, and annexin II was found on the surface of mesangial cells and in the mesangial matrix, co-localising with electron-dense deposits.

Conclusions Our data demonstrated an association between annexin II-binding IgG level and clinical/histological disease activity in proliferative lupus nephritis. Co-localization of annexin II with electron-dense deposits suggests a pathogenic role for annexin II.

\section{INCREASED URINARY HEPARANASE LEVELS ARE ASSOCIATED WITH ACTIVE LUPUS NEPHRITIS}

${ }^{1} \mathrm{CS}$ Cho, ${ }^{2} \mathrm{KJ}$ Kim, ${ }^{1} \mathrm{~B}$ In-Woon. ${ }^{7}$ Yeouido St. Mary's Hospital, Rheumatology, Seoul, Republic of Korea; ${ }^{2}$ St. Vincent's Hospital- The Catholic University of Korea, Rheumatology, Suwon, Republic of Korea

\subsection{6/lupus-2017-000215.206}

Background and aims Heparan sulfate in glomerular basement membrane is crucial for charge-selective filtration. Heparanase, an endoglycosidase that cleave heparan sulphate, is reported to be up-regulated in several proteinuric diseases. We investigated the association of urinary heparanase level with renal indices in patients with systemic lupus erythematosus (SLE).

Methods Urinary samples were collected from 76 patients with lupus nephritis (LN; 51 active and 25 inactive), 63 SLE patients without renal involvement and 28 healthy individuals (HC). Heparanase levels were measured by ELISA and normalised by urinary creatinine level $(\mathrm{mU} / \mathrm{mg})$.

Results Urinary heparanase levels were increased in SLE patients than HC $(\mathrm{p}<0.001)$. Patients with active LN had significantly higher urinary heparanase levels compared to patients with inactive $\mathrm{LN}$ and without renal involvement (both $\mathrm{p}<0.001$ ), however, there was no difference between latter groups. Urinary heparanase levels positively correlated with proteinuria (measured by spot urine protein/creatinine ratio) and renal SLEDAI $(\gamma=0.514, p<0.001$ and $\gamma=0.365$, $\mathrm{p}=0.004$, respectively), but inversely with serum C3 $(\gamma=-0.432, p<0.001), \mathrm{C} 4(\gamma=-0.279, \mathrm{p}=0.013)$, and $\mathrm{CH} 50$ levels $(\gamma=-0.336, p=0.003)$. In 39 patients with active $L N$ whose samples were obtained at the time of kidney biopsy, urinary heparanase levels showed positive correlation with activity index $(\gamma=0.409, p=0.011)$, but not with chronicity index $(\mathrm{p}>0.05)$. A cut-off value of $444 \mathrm{mU} / \mathrm{mg}$ predicted presence of active LN with sensitivity of $74.5 \%$ and specificity of $67.1 \%$.

Conclusions Urinary heparanase levels are increased in patients with active LN and reflect the activity of nephritis, indicating that urinary heparanase can serve as useful biomarker for active LN.

\section{SUBCLINICAL DETERIORATION OF LEFT VENTRICULAR DIASTOLIC FUNCTION IN SYSTEMIC LUPUS ERYTHEMATOSUS}

${ }^{1}$ YJ Choi, ${ }^{1}$ WH Yoo, ${ }^{1}$ WS Lee, ${ }^{2} \mathrm{C}$ Lee, ${ }^{2}$ MS Lee. ${ }^{1}$ Chonbuk National University Hospital, Internal Medicine, Jeonju, Republic of Korea; ${ }^{2}$ Wonkwang University Hospital, Internal Medicine, Iksan, Republic of Korea

\subsection{6/lupus-2017-000215.207}

Background and aims Systemic lupus erythematosus (SLE) represents diverse cardiac manifestation, but diastolic dysfunction has been reported infrequently. This study is aimed to 